\title{
Electrocardiographic J Wave and Cardiovascular Outcomes in the General Population (from the Atherosclerosis Risk In Communities Study)
}

Article in The American journal of cardiology · August 2016

DOI: 10.1016/j.amjcard.2016.06.047

CITATIONS

0

9 authors, including:

yi Wang

Duke University

2 PUBLICATIONS 5 CITATIONS

SEE PROFILE

\section{Edward Harvey Estes}

Duke University Medical Center 149 PUBLICATIONS $\quad 4,359$ CITATIONS

SEE PROFILE

\section{READS}

7
Larisa Tereshchenko

Oregon Health and Science University

103 PUBLICATIONS 405 CITATIONS

SEE PROFILE

\section{Elsayed Z Soliman}

Wake Forest School of Medicine 332 PUBLICATIONS 9,173 CITATIONS

SEE PROFILE 


\title{
Electrocardiographic J Wave and Cardiovascular Outcomes in the General Population (from the Atherosclerosis Risk In Communities Study)
}

\author{
Wesley T. O’Neal, MD, MPH ${ }^{\mathrm{a} *}$, Yi (Grace) Wang, $\mathrm{PhD}^{\mathrm{b}}$, Hau-Tieng Wu, MD, PhD ${ }^{\mathrm{c}}$, \\ Zhu-Ming Zhang, MD, MPH ${ }^{\mathrm{d}}$, Yabing Li, MD ${ }^{\mathrm{d}}$, Larisa G. Tereshchenko, MD, PhD ${ }^{\mathrm{e}, \mathrm{f}}$, \\ E. Harvey Estes, $\mathrm{MD}^{\mathrm{g}}$, Ingrid Daubechies, $\mathrm{PhD}^{\mathrm{b}}$, and Elsayed Z. Soliman, MD, MSc, MS ${ }^{\mathrm{d}, \mathrm{h}}$
}

\begin{abstract}
The association between the $\mathbf{J}$ wave, a key component of the early repolarization pattern, and adverse cardiovascular outcomes remains unclear. Inconsistencies have stemmed from the different methods used to measure the $\mathbf{J}$ wave. We examined the association between the $\mathbf{J}$ wave, detected by an automated method, and adverse cardiovascular outcomes in 14,592 (mean age $=54 \pm 5.8$ years; $56 \%$ women; $26 \%$ black) participants from the Atherosclerosis Risk In Communities (ARIC) study. The $\mathbf{J}$ wave was detected at baseline (1987 to 1989) and during follow-up study visits (1990 to 1992, 1993 to 1995, and 1996 to 1998) using a fully automated method. Sudden cardiac death, coronary heart disease death, and cardiovascular mortality were ascertained from hospital discharge records, death certificates, and autopsy data through December 31, 2010. A total of 278 participants (1.9\%) had evidence of a $J$ wave. Over a median follow-up of 22 years, 4,376 of the participants (30\%) died. In a multivariable Cox regression analysis adjusted for demographics, cardiovascular risk factors, and potential confounders, the $\mathbf{J}$ wave was not associated with an increased risk of sudden cardiac death (hazard ratio [HR] $0.74,95 \%$ CI 0.36 to 1.50 ), coronary heart disease death (HR $0.72,95 \%$ CI 0.40 to 1.32 ), or cardiovascular mortality (HR $1.16,95 \%$ CI 0.87 to 1.56 ). An interaction was detected for cardiovascular mortality by gender with men (HR 1.54, 95\% CI 1.09 to 2.19) having a stronger association than women (HR 0.74, 95\% CI 0.43 to 1.25 ; P-interaction $=0.030)$. In conclusion, our findings suggest that the $\mathbf{J}$ wave is a benign entity that is not associated with an increased risk for sudden cardiac arrest in middle-aged adults in the United States. (c) 2016 Elsevier Inc. All rights reserved. (Am J Cardiol 2016;118:811-815)
\end{abstract}

Early repolarization on the 12-lead electrocardiogram (ECG) has been traditionally considered a benign entity. ${ }^{1-4}$ However, recent reports have implicated early repolarization as a marker for adverse cardiovascular outcomes. ${ }^{5-8}$

\footnotetext{
${ }^{a}$ Division of Cardiology, Department of Medicine, Emory University School of Medicine, Atlanta, Georgia; ${ }^{\mathrm{b}}$ Department of Mathematics and Information Initiative, Duke University, Durham, North Carolina; ${ }^{\mathrm{c} D e-}$ partment of Mathematics, University of Toronto, Toronto, Ontario, Canada; ${ }^{\mathrm{d}}$ Department of Epidemiology and Prevention, Epidemiological Cardiology Research Center and hection on Cardiology, Department of Internal Medicine, Wake Forest School of Medicine, Winston-Salem, North Carolina; ${ }^{\mathrm{e} D e p a r t m e n t}$ of Cardiology, Johns Hopkins University School of Medicine, Baltimore, Maryland; ${ }^{\mathrm{f}}$ Knight Cardiovascular Institute, Oregon Health and Science University, Portland, Oregon; and ${ }^{\mathrm{g}}$ Department of Community and Family Medicine, Duke University Medical Center, Durham, North Carolina. Manuscript received April 9, 2016; revised manuscript received and accepted June 14, 2016.

Funding: The Atherosclerosis Risk in Communities study is carried out as a collaborative study supported by contracts HHSN268201100005C, HHSN268201100006C, HHSN268201100007C, HHSN268201100008C, HHSN268201100009C, HHSN268201100010C, HHSN268201100011C, and HHSN268201100012C from the National Heart, Lung, and Blood Institute.

See page 814 for disclosure information.

*Corresponding author: Tel: (+1) 404-727-2273

E-mail address: wesley.oneal@emory.edu (W.T. O'Neal).
}

Inherent in the definition of early repolarization are slurs and notches at the terminal portion of the QRS complex, which are collectively known as the "J wave." Hence, the prognostic significance of the $\mathbf{J}$ wave has become of great interest to clinical researchers. Although some reports have shown that the $\mathbf{J}$ wave is a benign finding, ${ }^{2,9,10}$ others have shown the opposite. ${ }^{11-14}$ Inconsistencies stem from different methods to measure the $\mathrm{J}$ wave, which have consisted of manual and partially automated techniques. Recently, a fully automated technique for J wave detection has been developed. ${ }^{15}$ The application of an entirely automated method to detect this component of early repolarization eliminates bias and enables researchers to compare the prognostic significance of this pattern. The purpose of this analysis was to examine the association between the $\mathrm{J}$ wave, detected by an automated method, and adverse cardiovascular outcomes in the Atherosclerosis Risk In Communities (ARIC) study.

\section{Methods}

ARIC prospectively enrolled 15,792 communitydwelling men and women between 45 and 64 years of age. Four field centers across the United States (Washington County, Maryland; Forsyth County, North Carolina; Jackson, Mississippi; suburban Minneapolis, Minnesota) 
recruited participants between 1987 and 1989. Participants returned for 4 follow-up examinations (1990 to 1992, 1993 to 1995,1996 to 1998 , and 2011 to 2013) and continue to be followed via annual telephone calls. End points are ascertained from hospital discharges that include any cardiovascular diagnoses from hospitals in the study communities. The study was approved by the institutional review boards at all participating universities, and all participants provided written informed consent at enrollment.

The following analysis aimed to examine the association of the $\mathrm{J}$ wave detected on the routine ECG with adverse cardiovascular outcomes. All participants with good quality ECG data and follow-up data were included. Participants were excluded if they had evidence of major ventricular conduction abnormalities (e.g., complete left or right bundle branch blocks), pacemakers, Wolf-Parkinson-White Syndrome or QRS duration $\geq 120 \mathrm{~ms}$, or if they reported a race other than black or white.

Digital 12-lead ECGs were obtained at baseline and at follow-up examinations using MAC PC ECG machines (Marquette Electronics, Milwaukee, Wisconsin). All ECGs were inspected for technical errors and adequate quality at the Epidemiology Coordinating and Research Center at the University of Alberta (Edmonton, Alberta, Canada) during the initial phases of the study and at the Epidemiological Cardiology Research Center at the Wake Forest School of Medicine (Winston-Salem, North Carolina) during later phases.

The J wave was detected at baseline (1987 to 1989) and during the first 3 study visits (1990 to 1992, 1993 to 1995 , and 1996 to 1998) as a time-dependent variable. J waves were defined as notches or slurs in the descending slope of the terminal portion of the QRS complex and were detected using a fully automated method described by Wang et al. ${ }^{15}$ Other electrocardiographic variables used in this analysis were heart rate, left ventricular hypertrophy, and J-point elevation. Left ventricular hypertrophy was defined by Cornell's voltage criteria. ${ }^{16} \mathrm{~J}$-point elevation was defined as a J-point amplitude of $\geq 0.1 \mathrm{mV}(1 \mathrm{~mm})$ in any lead. ${ }^{8}$

The primary outcome of this analysis was physicianadjudicated sudden cardiac death (SCD). In ARIC, fatal coronary heart disease (CHD) events that occurred before December 31, 2010 were reviewed. Death certificates, informant interviews, physician questionnaires, coroner reports, and hospital discharge summaries were reviewed to classify fatal CHD events as definite SCD, possible SCD, or non-SCD. SCD was defined as a sudden pulseless condition of cardiac origin in a previously stable individual. Details of potential cases were reviewed by physician adjudicators separately. Disagreement between reviewers was resolved by discussion. Cases of definite SCD were used in the present study. Secondary outcomes included CHD death and cardiovascular mortality. ${ }^{17}$ Cardiovascular mortality included the composite of fatal CHD, heart failure, and stroke events. Events occurring between the baseline examination and December 31, 2010 were included in this analysis.

Age, gender, and race were self-reported. Tobacco use was defined as current or former cigarette smoking. Diabetes was defined as a fasting glucose level $\geq 126 \mathrm{mg} / \mathrm{dl}$ (or nonfasting glucose $\geq 200 \mathrm{mg} / \mathrm{dl}$ ), a self-reported physician diagnosis of diabetes, or the use of diabetes medications. Systolic blood pressure was obtained from each participant using sphygmomanometers to measure 3 readings in the upright position after 5 minutes of rest. The average of the last 2 blood pressure measurements was used. Antihypertensive medication use was self-reported. Body mass index was defined as the weight in kilograms divided by the square of the height in meters. Low-density lipoprotein cholesterol levels were calculated indirectly using cholesterol values assayed from baseline serum samples. Prevalent CHD was defined by self-reported history of physiciandiagnosed myocardial infarction, coronary artery bypass surgery, coronary angioplasty, or electrocardiographic evidence of myocardial infarction.

Baseline characteristics for study participants were stratified by the presence of the $J$ wave. Categorical variables were reported as frequency and percentage, whereas continuous variables were recorded as mean \pm SD. Followup was defined as the time between the baseline visit until the outcome of interest, death, loss to follow-up, or end of follow-up (December 31, 2010). For J wave cases identified during subsequent study visits, follow-up time began at the time of $\mathbf{J}$ wave ascertainment. Cox regression was used to compute hazard ratios (HRs) and 95\% CIs for the association between the $\mathrm{J}$ wave and each outcome. Multivariable models were constructed as follows: model 1 adjusted for age, gender, and race; model 2 adjusted for model 1 covariates plus smoking, diabetes, systolic blood pressure, body mass index, low-density lipoprotein cholesterol, antihypertensive medications, left ventricular hypertrophy, CHD, and heart rate. Subgroup analyses by race, gender, and J-point elevation were performed, and tests for interaction were examined using model 2. Statistical significance for all comparisons, including interactions, was defined as $\mathrm{p}$ $<0.05$. SAS, version 9.4 (SAS Inc, Cary, North Carolina) was used for all analyses.

\section{Results}

A total of 14,592 (mean age $=54 \pm 5.8$ years; $56 \%$ women; $26 \%$ black) participants were included. There were 278 participants (1.9\%) who had evidence of a $\mathbf{J}$ wave. Of these, $126(45 \%)$ were detected on follow-up study visits. Baseline characteristics are listed in Table 1.

Over a median follow-up of 22 years, 4,376 participants (30\%) died. Of these, $503(11 \%)$ were attributed to SCD, 778 (18\%) were CHD deaths, and 2,303 (53\%) were due to cardiovascular disease. In a multivariable Cox regression model adjusted for demographics, cardiovascular risk factors, and potential confounders, the J wave was not associated with an increased risk of SCD, CHD death, or cardiovascular mortality (Table 2). An interaction was detected for cardiovascular mortality by gender, with men have a stronger association than women (Table 3). Interactions were not detected by race or J-point elevation (Table 3).

\section{Discussion}

In this analysis from ARIC, the presence of the $\mathrm{J}$ wave pattern, a key component of early repolarization, was not associated with an increased risk for SCD or CHD death. A 
Table 1

Baseline characteristics $(\mathrm{n}=14,592)$

\begin{tabular}{lccc}
\hline Characteristics & \multicolumn{2}{c}{ J wave } & \multirow{2}{*}{ P-value* } \\
\cline { 2 - 3 } & $\begin{array}{c}\text { Yes } \\
(\mathrm{n}=278)\end{array}$ & $\begin{array}{c}\text { No } \\
(\mathrm{n}=14,314)\end{array}$ & \\
& $54 \pm 5.9$ & $54 \pm 5.8$ & 0.67 \\
Age, mean \pm SD (years) & $132(47 \%)$ & $6,312(44 \%)$ & 0.26 \\
Men & $212(76 \%)$ & $3,601(25 \%)$ & $<0.0001$ \\
Black & $169(61 \%)$ & $8,329(58 \%)$ & 0.38 \\
Smoker & $29(10 \%)$ & $1,592(11 \%)$ & 0.72 \\
Diabetes mellitus & $138 \pm 42$ & $137 \pm 39$ & 0.83 \\
LDL cholesterol, & & & \\
$\quad$ mean \pm SD (mg/dl) & $28 \pm 4.9$ & $28 \pm 5.4$ & 0.72 \\
Body mass index, & & & \\
$\quad$ mean \pm SD (kg/m $\left.{ }^{2}\right)$ & $126 \pm 20$ & $121 \pm 19$ & $<0.0001$ \\
Systolic blood pressure, & & & \\
$\quad$ mean \pm SD (mm Hg) & $62 \pm 10$ & $67 \pm 10$ & $<0.0001$ \\
Heart rate, mean \pm SD (bpm) & $62(35 \%)$ & $4,224(30 \%)$ & 0.069 \\
Antihypertensive medications & $96(3.6 \%)$ & $627(4.4 \%)$ & 0.53 \\
Coronary heart disease & $10(3.6 \%)$ & 0.049 \\
Left ventricular hypertrophy & $1(0.4 \%)$ & $290(2.0 \%)$ & \\
J-point elevation & $117(42 \%)$ & $1,669(12 \%)$ & $<0.0001$ \\
\hline
\end{tabular}

$\mathrm{Bpm}=$ beats per minute $\mathrm{LDL}=$ low-density lipoprotein $\mathrm{SD}=$ standard deviation.

* Statistical significance for categorical data was tested using the chisquare procedure, and continuous data were tested using the Student $t$ test.

significant association was observed between the $\mathbf{J}$ wave and cardiovascular mortality in men but not women. Overall, our findings suggest that the $\mathbf{J}$ wave is a benign entity that is not associated with an increased risk for sudden cardiac arrest in middle-aged, white and black adults in the United States. However, the $\mathbf{J}$ wave possibly has prognostic significance for men regarding cardiovascular mortality.

The association between early repolarization and adverse cardiovascular outcomes remains controversial. A casecontrol study of 206 subjects who were resuscitated after cardiac arrest due to idiopathic ventricular fibrillation and 412 matched controls showed that early repolarization (QRS-ST junction elevation $\geq 0.1 \mathrm{mV}$ ) was more frequent in case subjects than controls. ${ }^{\frac{5}{5}}$ Another case-control study of 45 patients with idiopathic ventricular fibrillation showed that J-point elevation (defined as any or $\geq 0.1 \mathrm{mV}$ elevation) was more prevalent in cases than controls. ${ }^{7}$ A follow-up study of 10,864 middle-aged subjects from the Social Insurance Institution's Coronary Heart Disease Study in Finland also has shown that J-point elevation $(\geq 0.1 \mathrm{mV})$ is associated with an increased risk of cardiac death (relative risk $=1.28,95 \%$ CI 1.04 to 1.59 ), and the risk of death markedly increases with elevation $\geq 0.2 \mathrm{mV}$ (relative risk $=2.98,95 \% \mathrm{CI}=1.85$ to 4.92 ). ${ }^{6}$ In addition, data from ARIC have shown that J-point elevation $\geq 0.1 \mathrm{mV}$ is associated with an increased risk of SCD in whites (HR 2.03, 95\% CI 1.28 to 3.21 ) and in women (HR 2.54, 95\% CI 1.34, 4.82). ${ }^{8}$ However, an analysis of 2,234 ECGs from a sample of adults in Oakland, California found that early repolarization (J-point elevation $\geq 0.1 \mathrm{mV}$ ) was not associated with an increased risk for cardiovascular mortality. Similarly, patients from the Veterans Affairs Palo Alto Health Care System $^{2,3}$ and the Coronary Artery Risk Development in Young Adults Study have failed to show an association between J-point elevation and adverse cardiac outcomes. ${ }^{4}$
Table 2

Risk of adverse cardiovascular events $(n=14,592)$

\begin{tabular}{lccc}
\hline Model & $\begin{array}{c}\text { Sudden Cardiac Death } \\
\text { HR }(95 \% \mathrm{CI})\end{array}$ & $\begin{array}{c}\text { CHD Death } \\
\text { HR }(95 \% \mathrm{CI})\end{array}$ & $\begin{array}{c}\text { Cardiovascular } \\
\text { Mortality } \\
\text { HR }(95 \% \mathrm{CI})\end{array}$ \\
\hline Model 1* & $0.59(0.29,1.19)$ & $0.56(0.31,1.01)$ & $0.93(0.70,1.24)$ \\
Model $2^{\dagger}$ & $0.74(0.36,1.50)$ & $0.72(0.40,1.32)$ & $1.16(0.87,1.56)$ \\
\hline
\end{tabular}

$\mathrm{CHD}=$ coronary heart disease $\mathrm{CI}=$ confidence interval; $\mathrm{HR}=$ hazard ratio.

* Adjusted for age, gender, and race.

${ }^{\dagger}$ Adjusted for model 1 covariates plus smoking, diabetes, systolic blood pressure, body mass index, low-density lipoprotein cholesterol, antihypertensive medications, left ventricular hypertrophy, coronary heart disease, and heart rate.

Reports also are conflicting regarding the prognostic significance of the $\mathbf{J}$ wave. A case-control study of 21 athletes with SCD showed that the J wave and/or QRS slurring without ST elevation in the inferior and lateral leads were more frequently recorded in cases than controls $(28.6 \%$ vs $7.9 \%, \mathrm{p}=0.007) .{ }^{11}$ A comparison of the ECGs between those with idiopathic ventricular fibrillation and controls demonstrated that QRS notching was significantly more prevalent among cases than among controls. ${ }^{12}$ A casecontrol study also found that the $\mathrm{J}$ wave was more common in patients with a history of idiopathic ventricular fibrillation (odds ratio 4.0, 95\% CI 2.0 to 7.9 ) ${ }^{13}$ In addition, the highest risk for all-cause and cardiovascular mortalities was observed with early repolarization with terminal QRS "notching" in subjects from the third French Monitoring Trends and Determinants in Cardiovascular Disease Project. ${ }^{14}$ In contrast, reports from the Veterans Affairs Palo Alto Health Care System ${ }^{2,9}$ and a study of Italian Olympic athletes have failed to show that the $\mathbf{J}$ wave is a malignant electrocardiographic finding. ${ }^{10}$

Our findings from a large, biracial cohort study do not support an association of the $\mathbf{J}$ wave with an increased risk for SCD or acute CHD death. Most studies which support that early repolarization and the $\mathrm{J}$ wave are associated with adverse cardiovascular outcomes are from case-control studies of participants with idiopathic ventricular fibrillation. ${ }^{5-7,11-13}$ In contrast, negative studies are predominately from longitudinal registries. ${ }^{1-4,9,10}$ The different study designs used is a possible explanation for the inconsistent results. In addition, the techniques to identify each electrocardiographic entity and the definitions of early repolarization and $\mathbf{J}$ wave varied greatly between studies. Potentially, our findings provide clarity regarding the prognostic significance of the $\mathbf{J}$ wave, as we relied on an entirely automated technique with visual confirmation by trained electrocardiographers. ${ }^{15}$ Finally, our study population included participants with cardiovascular disease risk factors and CHD. Most studies have linked early repolarization to idiopathic ventricular fibrillation rather than SCD due to underlying CHD. Therefore, it is possible that the population examined was quite different than subjects with idiopathic events, as ARIC participants would be more likely to develop SCD due to underlying atherosclerosis. Our findings do support an association between the J-wave pattern and cardiovascular mortality in men. Although the 
Table 3

Subgroup analyses $(n=14,592)$

\begin{tabular}{|c|c|c|}
\hline Outcome & $\mathrm{HR}(95 \% \mathrm{CI})^{*}$ & P-Interaction \\
\hline \multicolumn{3}{|c|}{ Sudden Cardiac Death } \\
\hline \multicolumn{3}{|c|}{ Race } \\
\hline White & $0.62(0.09,4.44)$ & 0.83 \\
\hline Black & $0.73(0.34,1.57)$ & \\
\hline \multicolumn{3}{|l|}{ Sex } \\
\hline Male & $1.21(0.59,2.48)$ & - \\
\hline Female & - & \\
\hline \multicolumn{3}{|c|}{ J-Point Elevation } \\
\hline No & $0.40(0.10,1.61)$ & 0.35 \\
\hline Yes & $1.07(0.45,2.50)$ & \\
\hline \multicolumn{3}{|l|}{ CHD Death } \\
\hline \multicolumn{3}{|l|}{ Race } \\
\hline White & $0.80(0.20,3.21)$ & 0.89 \\
\hline Black & $0.69(0.35,1.34)$ & \\
\hline \multicolumn{3}{|l|}{ Sex } \\
\hline Male & $1.06(0.56,2.00)$ & 0.090 \\
\hline Female & $0.17(0.02,1.24)$ & \\
\hline \multicolumn{3}{|c|}{ J-Point Elevation } \\
\hline No & $0.75(0.31,1.83)$ & 0.76 \\
\hline Yes & $0.73(0.32,1.69)$ & \\
\hline \multicolumn{3}{|c|}{ Cardiovascular Mortality } \\
\hline \multicolumn{3}{|c|}{ Race } \\
\hline White & $1.24(0.66,2.31)$ & 0.85 \\
\hline Black & $1.10(0.79,1.53)$ & \\
\hline \multicolumn{3}{|l|}{ Sex } \\
\hline Male & $1.54(1.09,2.19)$ & 0.030 \\
\hline Female & $0.74(0.43,1.25)$ & \\
\hline \multicolumn{3}{|c|}{ J-Point Elevation } \\
\hline No & $1.12(0.74,1.69)$ & 0.85 \\
\hline Yes & $1.21(0.80,1.84)$ & \\
\hline
\end{tabular}

$\mathrm{CHD}=$ coronary heart disease $\mathrm{CI}=$ confidence interval; $\mathrm{HR}=$ hazard ratio.

* Adjusted for age, gender, race, smoking, diabetes, systolic blood pressure, body mass index, low-density lipoprotein cholesterol, antihypertensive medications, left ventricular hypertrophy, coronary heart disease, and heart rate.

reason for this finding is unclear, further research is needed to determine the prognostic significance of this association. Nonetheless, our findings support that the $\mathbf{J}$ wave is not associated with an increased risk for SCD or acute CHD death in the general population, and further research is needed due to these inconsistent reports.

Given that most studies showing a positive association between early repolarization and SCD have come from casecontrol studies of survivors of idiopathic ventricular fibrillation, it is possible that early repolarization merits further investigation in the appropriate setting. For example, subjects who have early repolarization with concerning clinical histories (e.g., syncope or a family history of SCD) possibly will benefit from further cardiac work-up. However, the data presented in this analysis and others with null findings do not support an association between features of early repolarization in which the $\mathrm{J}$ wave is a key component and SCD. This suggests that widespread screening and implementing preventive therapies in subjects who have these patterns without high-risk features is unwarranted.

The present study should be interpreted in the context of several limitations. Although we ascertained deaths by review of hospital discharge records and autopsy reports, it is possible that events were misclassified. Several baseline covariates were self-reported and potentially subjected our analysis to recall bias. Furthermore, we included several covariates in our multivariable models that likely influenced the outcomes of interest, but we acknowledge that residual confounding remains a possibility.

Acknowledgment: The authors thank the staff and participants of the ARIC study for their important contributions.

\section{Disclosures}

The authors have no conflicts of interest to disclose.

1. Klatsky AL, Oehm R, Cooper RA, Udaltsova N, Armstrong MA. The early repolarization normal variant electrocardiogram: correlates and consequences. Am J Med 2003;115:171-177.

2. Uberoi A, Jain NA, Perez M, Weinkopff A, Ashley E, Hadley D, Turakhia MP, Froelicher V. Early repolarization in an ambulatory clinical population. Circulation 2011;124:2208-2214.

3. Perez MV, Uberoi A, Jain NA, Ashley E, Turakhia MP, Froelicher V. The prognostic value of early repolarization with ST-segment elevation in African Americans. Heart Rhythm 2012:9:558-565.

4. Ilkhanoff L, Soliman EZ, Prineas RJ, Walsh JA 3rd, Ning H, Liu K, Carr JJ, Jacobs DR Jr, Lloyd-Jones DM. Clinical characteristics and outcomes associated with the natural history of early repolarization in a young, biracial cohort followed to middle age: the Coronary Artery Risk Development in Young Adults (CARDIA) study. Circ Arrhythm Electrophysiol 2014;7:392-399.

5. Haissaguerre M, Derval N, Sacher F, Jesel L, Deisenhofer I, de Roy L, Pasquie JL, Nogami A, Babuty D, Yli-Mayry S, De Chillou C, Scanu P, Mabo P, Matsuo S, Probst V, Le Scouarnec S, Defaye P, Schlaepfer J, Rostock T, Lacroix D, Lamaison D, Lavergne T, Aizawa Y, Englund A, Anselme F, O'Neill M, Hocini M, Lim KT, Knecht S, Veenhuyzen GD, Bordachar P, Chauvin M, Jais P, Coureau G, Chene G, Klein GJ, Clementy J. Sudden cardiac arrest associated with early repolarization. $N$ Engl J Med 2008;358: 2016-2023.

6. Tikkanen JT, Anttonen O, Junttila MJ, Aro AL, Kerola T, Rissanen HA, Reunanen A, Huikuri HV. Long-term outcome associated with early repolarization on electrocardiography. $N$ Engl J Med 2009;361:2529-2537.

7. Rosso R, Kogan E, Belhassen B, Rozovski U, Scheinman MM, Zeltser D, Halkin A, Steinvil A, Heller K, Glikson M, Katz A, Viskin S. J-point elevation in survivors of primary ventricular fibrillation and matched control subjects: incidence and clinical significance. J Am Coll Cardiol 2008;52:1231-1238.

8. Olson KA, Viera AJ, Soliman EZ, Crow RS, Rosamond WD. Longterm prognosis associated with J-point elevation in a large middle-aged biracial cohort: the ARIC study. Eur Heart J 2011;32:3098-3106.

9. Muramoto D, Yong CM, Singh N, Aggarwal S, Perez M, Ashley E, Hadley D, Froelicher V. Patterns and prognosis of all components of the J-wave pattern in multiethnic athletes and ambulatory patients. Am Heart J 2014;167:259-266.

10. Quattrini FM, Pelliccia A, Assorgi R, DiPaolo FM, Squeo MR, Culasso F, Castelli V, Link MS, Maron BJ. Benign clinical significance of J-wave pattern (early repolarization) in highly trained athletes. Heart Rhythm 2014;11:1974-1982.

11. Cappato R, Furlanello F, Giovinazzo V, Infusino T, Lupo P, Pittalis M, Foresti S, De Ambroggi G, Ali H, Bianco E, Riccamboni R, Butera G, Ricci C, Ranucci M, Pelliccia A, De Ambroggi L. J wave, QRS slurring, and ST elevation in athletes with cardiac arrest in the absence of heart disease: marker of risk or innocent bystander? Circ Arrhythm Electrophysiol 2010;3:305-311.

12. Merchant FM, Noseworthy PA, Weiner RB, Singh SM, Ruskin JN, Reddy VY. Ability of terminal QRS notching to distinguish benign from malignant electrocardiographic forms of early repolarization. Am J Cardiol 2009;104:1402-1406. 
13. Rosso R, Glikson E, Belhassen B, Katz A, Halkin A, Steinvil A, Viskin S. Distinguishing "benign" from "malignant early repolarization": the value of the ST-segment morphology. Heart Rhythm 2012;9: 225-229.

14. Rollin A, Maury P, Bongard V, Sacher F, Delay M, Duparc A, Mondoly P, Carrie D, Ferrieres J, Ruidavets JB. Prevalence, prognosis, and identification of the malignant form of early repolarization pattern in a population-based study. Am J Cardiol 2012;110:1302-1308.

15. Wang YG, Wu HT, Daubechies I, Li Y, Estes EH, Soliman EZ. Automated J wave detection from digital 12-lead electrocardiogram. J Electrocardiol 2015;48:21-28.
16. Devereux RB, Casale PN, Eisenberg RR, Miller DH, Kligfield P. Electrocardiographic detection of left ventricular hypertrophy using echocardiographic determination of left ventricular mass as the reference standard. Comparison of standard criteria, computer diagnosis and physician interpretation. J Am Coll Cardiol 1984;3: $82-87$.

17. Williams JE, Massing M, Rosamond WD, Sorlie PD, Tyroler HA. Racial disparities in CHD mortality from 1968-1992 in the state economic areas surrounding the ARIC study communities. Atherosclerosis Risk in Communities. Ann Epidemiol 1999;9: $472-480$. 